\title{
Semi-Infinite Transportation Problems
}

\author{
H. Voigt
}

\begin{abstract}
An old set partitioning problem is treated as a special case of the Kantorovic Monge transportation problem. This problem is then related to Klötzler's transportation flow problems which allow the consideration of a local cost rate, instead of the constant cost rate in the Kantorovič-Monge problem. Three possibilities for the numerical solution of the problem are discussed and briefly compared.
\end{abstract}

Keywords: Transportation problem, Kantorovič-Monge problem, transportation flow problem, set partitioning, market area problem

AMS subject classification: $90 \mathrm{C} 34,49 \mathrm{~N} 15,90 \mathrm{~B} 10$

\section{Introduction}

We study an old problem which is known as a set partitioning problem or market area problem. The economical background may be described as follows: In a region $\bar{\Omega}$ there are $m$ stocks (supply points) $T_{1}, \ldots, T_{m}$ for a certain product each with a positive supply $a_{1}, \ldots, a_{m}$. The demand on the product is described by a density function $b(\cdot)$ such that the total demand in $\bar{\Omega}$ and the total supply are equal. (The demand of a subset $e$ of $\bar{\Omega}$ is given by $\int_{e} b(t) d t$.) The problem is to partition $\bar{\Omega}$ into $m$ districts such that the demand of the $i$-th district $\Omega_{i}$ is satisfied from the $i$-th stock and the overall transportation effort (measured by the Euclidean distance) is minimal.

This leads to a non-standard optimization problem: Find a partition of $\bar{\Omega}$ into non-overlapping subsets $\Omega_{i} \quad(i=1, \ldots, m)$ such that

$$
\sum_{i=1}^{m} \int_{\Omega_{i}}\left|T_{i}-t\right| d t
$$

is minimal and the restrictions

$$
\int_{\Omega_{i}} b(t) d t=a_{i} \quad(i=1, \ldots, \dot{m})
$$

are satisfied. $\left(|\cdot|\right.$ denotes the Euklidean norm in $\mathbb{E}^{2}$.) This problem appeared in similar form in [1] as regional design problem and in [7] as generalized market area problem.

In this paper we shall analyze this problem in the context of the Kantorovič-Monge transportation problem (cf. [3]). The main result is that the required partitioning is

H. Voigt: Univ. Leipzig, Institut für Mathematik, Augustusplatz 10, D - 04109 Leipzig 
a consequence of optimality in the transportation problem. This is discussed in the next section. In Section 3, we compare the transportation problem with Klötzler's transportation flow problem (cf. [4]). This allows us to reformulate the problem in a more realistic manner: the transportation cost rate may depend on the location. Finally, in Section 4, we present three approaches to the practical solution of the transportation flow problem. In the first and second approaches, the problem is partially dicretized which leads to a semi-infinite linear or a nonlinear program. This can subsequently be solved by a simplex technique and a nonlinear programming method, respectively. In the third approach, the problem is totally discretized which leads to a flow problem in a graph. These approaches are briefly compared.

\section{The semi-infinite transportation problem}

Omitting the set partition requirement from problem (1.1)-(1.2), we can reformulate this as a special Kantorovič-Monge problem. The general problem formulation is as follows:

Minimize

$$
\int_{\overline{\mathbf{\Omega}} \times \overline{\mathbf{\Omega}}}\left|t-t^{\prime}\right| d \psi\left(t, t^{\prime}\right)
$$

subject to

$$
\psi \in C^{*}(\bar{\Omega} \times \bar{\Omega}), \quad \psi \geq 0
$$

and

$$
\begin{gathered}
\psi(e, \bar{\Omega})=\phi_{0}(e) \quad(e \in \mathfrak{B}) \\
\psi\left(\bar{\Omega}, e^{\prime}\right)=\phi_{1}\left(e^{\prime}\right) \quad\left(e^{\prime} \in \mathfrak{B}\right) .
\end{gathered}
$$

Here $\bar{\Omega}$ is a compact subset of the Euclidean space $\mathbb{E}^{2}, \mathfrak{B}$ is the $\sigma$-Algebra of all Lebesgue measurable subsets of $\bar{\Omega}$, and $\phi_{0}, \phi_{1}$ are given non-negative measures on $\bar{\Omega}$ satisfying

$$
\phi_{0}(\bar{\Omega})=\phi_{1}(\bar{\Omega})>0
$$

The measures $\phi_{0}, \phi_{1}$ describe the distribution of supply and demand over $\bar{\Omega}$ whereas the variable $\psi$ describes the "translocation of masses": $\psi\left(e, e^{\prime}\right)$ is the quantity of the product moved from one subset $e$ to another subset $e^{\prime}$ of $\bar{\Omega}$. The constraints (2.3)-(2.4) reflect the requirement that the total supply of a subset $e$ must be moved somewhere and that the total demand of a subset $e^{\prime}$ must be satisfied from somewhere. The objective functional (2.1) is the total effort necessary to realize the transportation plan $\psi$.

The dual problem to (2.1)-(2.4) according to Kantorovič and Rubinštein is:

Maximize

$$
\int_{\tilde{\Omega}} u(t) d \phi_{0}(t)+\int_{\bar{\Omega}} v\left(t^{\prime}\right) d \phi_{1}\left(t^{\prime}\right)
$$

subject to

$$
u, v \in C(\bar{\Omega})
$$


and

$$
u(t)+v\left(t^{\prime}\right) \leq\left|t-t^{\prime}\right| \quad\left(t, t^{\prime} \in \vec{\Omega}\right) .
$$

For a feasible solution $\psi$ to problem (2.1)-(2.4) we shall say that a transport from $t \in \bar{\Omega}$ to $t^{\prime} \in \bar{\Omega}$ occurs if for any neighbourhood $U$ of $t$ and any neighbourhood $U^{\prime}$ of $t^{\prime}$ the value $\psi\left(U, U^{\prime}\right)$ is positive. This is symbolized with $t \stackrel{\psi}{\longrightarrow} t^{\prime}$.

The Kantorovič-Rubinštein duality theorem (cf. [8]) states that both problems (2.1)(2.4) and (2.6)-(2.8) have optimal solutions with equal optimal values. Moreover, a feasible solution $\psi$ to problem (2.1)-(2.4) is optimal if and only if there is a feasible solution $(u, v)$ to problem (2.6)-(2.8) such that

$$
u(t)+v\left(t^{\prime}\right)=\left|t-t^{\prime}\right| \quad \text { if } t \stackrel{\psi}{\longrightarrow} t^{\prime}
$$

This is exactly the complementary slackness condition from linear programming.

In this strong form the duality theorem holds only if the integrand in (2.1) is the distance in a compact metric space. If it is replaced with an arbitrary non-negative continuous function the existence of an optimal solution to problem (2.6)-(2.8) is not guaranteed (cf. [6]).

The classical Hitchcock problem is derived from problem (2.1)-(2.4) if both $\phi_{0}$ and $\phi_{1}$ are finitely-discrete measures. To obtain the model described in the introduction (without the set partition condition) we have to specify $\phi_{0}$ as a discrete measure concentrated at the finite set $\left\{T_{1}, \ldots, T_{m}\right\}$ with masses $a_{1}, \ldots, a_{m}$ :

$$
\phi_{0}=\sum_{i} a_{i} \delta T_{i}
$$

( $\delta_{t}$ denotes the Dirac measure concentrated at $t$ ) and $\phi_{1}$ as an absolutely continuous measure with density $b$ :

$$
d \phi_{1}=b d t \text {. }
$$

Since here only one of the measures $\phi_{0}, \phi_{1}$ is finite we call this a semi-infinite trans. portation problem.

It is now a natural idea to introduce measures $\psi_{1}, \ldots, \psi_{m}$ that describe the translocation of the supply in $T_{1}, \ldots, T_{m}$, respectively. For a feasible solution $\psi$ to problem (2.1)-(2.4) we set

$$
\psi_{i}\left(e^{\prime}\right)=\psi\left(\left\{T_{i}\right\}, e^{\prime}\right) \quad\left(i=1, \ldots, m ; e^{\prime} \in \mathfrak{B}\right) .
$$

From (2.2) and (2.3) we then obtain

$$
\psi_{i} \in C^{*}(\bar{\Omega}), \psi_{i} \geq 0 \quad(i=1, \ldots, m)
$$

and

$$
\psi_{i}(\bar{\Omega})=\phi_{0}\left(\left\{T_{i}\right\}\right)=a_{i} \quad(i=1, \ldots, m) .
$$

For $e_{0}=\left\{T_{1}, \ldots, T_{m}\right\}$ and arbitrary $e^{\prime} \in \mathfrak{B}$ we have

$$
\psi\left(\bar{\Omega} \backslash e_{0}, e^{\prime}\right) \leq \psi\left(\bar{\Omega} \backslash e_{0}, \bar{\Omega}\right)=\phi_{0}\left(\bar{\Omega} \backslash e_{0}\right)=0
$$


and, therefore,

$$
\sum_{i=1}^{m} \psi_{i}\left(e^{\prime}\right)=\psi\left(e_{0}, e^{\prime}\right)=\psi\left(\bar{\Omega}, e^{\prime}\right)=\phi_{1}\left(e^{\prime}\right) \quad\left(e^{\prime} \in \mathfrak{B}\right) .
$$

Conversely, if the measures $\psi_{1}, \ldots, \psi_{m}$ satisfy (2.13)-(2.15), then, setting

$$
\psi\left(e, e^{\prime}\right)=\sum_{i=1}^{m} \delta_{T_{i}}(e) \psi_{i}\left(e^{\prime}\right)
$$

we obtain a measure $\psi$ satisfying (2.1)-(2.4): For arbitrary $e, e^{\prime} \in \mathfrak{B}$ it follows

$$
\begin{aligned}
\psi(e, \bar{\Omega}) & =\sum_{i} \delta_{T_{i}}(e) \psi_{i}(\bar{\Omega})=\sum_{i} \delta_{T_{i}} a_{i}=\phi_{0}(e) \\
\psi\left(\bar{\Omega}, e^{\prime}\right) & =\sum_{i} \delta_{T_{i}}(\ddot{\Omega}) \psi_{i}\left(e^{\prime}\right)=\sum_{i} \psi_{i}\left(e^{\prime}\right)=\phi_{1}\left(e^{\prime}\right)
\end{aligned}
$$

Since the measure $\phi_{1}$ is assumed to be absolutely continuous the measures $\psi_{\mathrm{i}}$ are absolutely continuous, too. This follows from $\psi_{i}\left(e^{\prime}\right) \leq \phi_{1}\left(e^{\prime}\right)$ for $i=1, \ldots, m$ and $e^{\prime} \in \mathfrak{B}$ (which is an immediate consequence of (2.13) and (2.15)). So, according to the RadonNikodym theorem, the measures $\psi_{i}$ have representations

$$
d \psi_{i}=b_{i} d t \quad(i=1, \ldots, m)
$$

with densities $b_{i} \in L_{1}(\bar{\Omega})$. These densities then have the obvious properties

$$
b_{i}\left(t^{\prime}\right) \geq 0 \quad \text { a.e. in } \bar{\Omega}
$$

and

$$
\begin{array}{cc}
\int_{\bar{\Omega}} b_{i}\left(t^{\prime}\right) d t^{\prime}=a_{i} \quad(i=1, \ldots, m) \\
\sum_{i=1}^{m} b_{i}\left(t^{\prime}\right)=b\left(t^{\prime}\right) \quad \text { a.e. in } \bar{\Omega}
\end{array}
$$

and from (2.18) and (2.20) we further get $b_{i} \in L_{\infty}(\bar{\Omega})$.

We can now formulate the semi-infinite transportation problem in terms of the measures $\psi_{1}, \ldots, \psi_{m}$ and in terms of the densities $b_{1}, \ldots, b_{m}$ as well. To do this we have to substitute first the representation (2.16) and then (2.17) into (2.1) to obtain the objective functional in terms of the $\psi_{i}$ and the $b_{i}$, respectively. This leads to the following problems:

Minimize

$$
\sum_{i=1}^{m} \int_{\tilde{\Omega}}\left|T_{i^{\prime}}-t^{\prime}\right| d \psi_{i}\left(t^{\prime}\right)
$$


subject to (2.13)-(2.15) and

Minimize

$$
\sum_{i=1}^{m} \int_{\bar{\Omega}}\left|T_{i}-t^{\prime}\right| b_{i}\left(t^{\prime}\right) d t^{\prime}
$$

subject to $(2.18)-(2.20)$.

In a similar way we may reformulate the dual problem (2.6)-(2.8) to get the following dual problem to $(2.22),(2.18)-(2.20)$ :

Maximize

$$
\sum_{i=1}^{m} a_{i} u\left(T_{i}\right)+\int_{\delta} b\left(t^{\prime}\right) v\left(t^{\prime}\right) d t^{\prime}
$$

subject to

$$
u\left(T_{i}\right)+v\left(t^{\prime}\right) \leq\left|T_{i}-t^{\prime}\right| \quad\left(t^{\prime} \in \bar{\Omega}\right) .
$$

Since only the values $u\left(T_{i}\right)$ are significant we can replace the function $u$ in the dual problem with a vector $\left(u_{1}, \ldots, u_{m}\right)^{\top} \in \mathbb{R}^{m}$ and get the following final formulation for the dual problem:

Maximize

$$
\sum_{i=1}^{m} a_{i} u_{i}+\int_{\Omega} b\left(t^{\prime}\right) v\left(t^{\prime}\right) d t^{\prime}
$$

subject to

$$
\left(u_{1}, \ldots, u_{m}\right)^{\top} \in \mathbb{R}^{m}, v \in C(\bar{\Omega})
$$

and

$$
u_{i}+v\left(t^{\prime}\right) \leq\left|T_{i}-t^{\prime}\right| \quad\left(t^{\prime} \in \bar{\Omega}\right) .
$$

From the Kantotovič-Rubinštein duality theorem we obtain now the following optimality conditions in terms of the measures $\psi_{i}$ and the densities $b_{i}$, respectively:

Proposition 1. A set of measures $\psi_{1}, \ldots, \psi_{m}$ satisfying (2.13)-(2.15) (of functions $b_{1}, \ldots, b_{m}$ satisfying (2.18)-(2.20)) is optimal if and only if there exist a vector $u$ and $a$ function $v$ satisfying (2.24)-(2.25) such that

$$
u_{i}+v\left(t^{\prime}\right)=\left|T_{i}-t^{\prime}\right| \quad \text { if } T_{i} \stackrel{\psi_{i}}{\longrightarrow} t^{\prime} .
$$

Here $T_{i} \stackrel{\psi_{i}}{\longrightarrow} t^{\prime}$ means that $\psi_{i}\left(U^{\prime}\right)>0$ for any neighbourhood $U^{\prime}$ of $t^{\prime}$.

This optimality condition allows a conclusion about the structure of any optimal solution. Let $\psi_{1}, \ldots, \psi_{m}$ be an optimal solution to (2.21), (2.13)-(2.15) and assume that for some $t^{\prime} \in \bar{\Omega}$ both $T_{i} \stackrel{\psi_{i}}{\longrightarrow} t^{\prime}$ and $T_{j} \stackrel{\psi_{j}}{\longrightarrow} t^{\prime}$ (for $i \neq j$ ) hold. Subtracting the respective conditions $(2.26)$ we get

$$
\left|T_{i}-t^{\prime}\right|-\left|T_{j}-t^{\prime}\right|=u_{i}-u_{j}
$$

But this means that all such points $t^{\prime}$ are located on a hyperbola with focusses $T_{i}$ and $T_{j}$ and a real axis. of length $\left|u_{i}-u_{j}\right|$. Thus, if we denote by $\Omega_{i}$ the set of all points $t^{\prime} \in \bar{\Omega}$ for which $T_{i} \stackrel{\psi_{i}}{\longrightarrow} t^{\prime}$ holds (this is actually the support of $\psi_{i}$ ), then we get the following result: 
Proposition 2. For any optimal solution $\psi_{1}, \ldots, \psi_{m}$ of problem (2.21), (2.13)(2.15), the sets $\Omega_{i}$ are pairwise non-overlapping and the boundaries of these sets are. contained in the hyperbolas defined by (2.27).

Finally, from (2.26) we obtain

$$
v\left(t^{\prime}\right)=u_{i}-\left|T_{i}-t^{\prime}\right| \quad \text { for } t^{\prime} \in \Omega_{i}
$$

i.e. the graph of $v$ is composed of cones with vertices in $T_{i}$ at height $u_{i}$ and unit inclination.

The densities $b_{i}$ at an optimal solution to problem (2.22), (2.18)-(2.20) then have the form

$$
b_{i}\left(t^{\prime}\right)= \begin{cases}b\left(t^{\prime}\right) & \text { if } t^{\prime} \in \Omega_{i} \\ 0 & \text { else. }\end{cases}
$$

For almost all $t^{\prime} \in \bar{\Omega}$, the vector $\left(b_{1}\left(t^{\prime}\right), \ldots, b_{m}\left(t^{\prime}\right)\right)^{\top}$ is an extreme point of the polyhedron

$$
\left\{\beta \in \mathbb{R}^{m}: \beta_{i} \geq 0 \text { and } \sum_{i} \beta_{i}=b\left(t^{\prime}\right)\right\} .
$$

It is well known from the theory of capacitated linear programs (cf. [9]) that this property characterizes the extreme points of the feasible set defined by (2.18)-(2.20). The only difference is that in our setting the capacitating polyhedron is not constant.

For to the problem (1.1)-(1.2) we get now the following final result:

Any optimal solution of problem (2.21), (2.13)-(2.15) has according to Proposition 2 the required set partition property and hence is an optimal solution of problem (1.1)(1.2). The districts are the supports of the measures $\psi_{i}$ at the optimal solution.

It should be noted that in this context the assumption $T_{i} \in \bar{\Omega}$ (made in the verbal introduction of the problem) is not really necessary. All remains true if supply points are outside the region.

\section{The transportation flow problem}

During the last years Klötzler developed his concept of transportation flow problems (cf. [4]). It is based on the ideas of Kantorovič and Rubinštein but goes an essential step beyond their model. In the objective functional (2.1) of the Kantorović-Monge problem above a constant (i.e. location independent) transportation cost rate is implicitely assumed such that all transports go along straight lines (not necessarily within the region) and a transportation path or trajectory does not even occur in the model. Klötzler replaced this with a local transportation cost rate which may depend both on the location and the direction of transport. His general problem is formulated as follows:

Minimize

$$
\int_{\Omega} r(t, d \mu(t))
$$

subject to

$$
\mu \in L_{\infty}^{2}(\Omega)^{*}
$$


and

$$
\int_{\Omega} \nabla^{\top} \sigma(t) d \mu(t)=\int_{\Omega} \sigma(t) d \alpha(t) \quad \text { for all } \sigma \in W_{\infty}^{1}(\Omega)
$$

Here $\Omega$ is a bounded strongly Lipschitz domain in $\mathbb{E}^{2}$, and $\alpha$ is a (signed) measure on the $\sigma$-Algebra $\mathfrak{B}$ of all Lebesgue measurable subsets of $\Omega$ such that

$$
\int_{\Omega} d \alpha=0
$$

The real function $r$ on $\Omega \times \mathbb{E}^{2}$ is assumed to have the following properties:

- $r(\cdot, w)$ is summable on $\Omega$ for all $w \in \mathbb{E}^{2}$.

- $r(t, \cdot)$ is positive homogeneous of degree one and convex on $\mathbb{E}^{2}$ for all $t \in \Omega$.

- $\gamma_{1}|w| \leq r(t, w) \leq \gamma_{2}|w|$ for all $t \in \Omega$ and $w \in \mathbb{E}^{2}$ with some positive constants $\gamma_{1}, \gamma_{2}$.

The objective functional is defined by

$$
\int_{\Omega} r(t, d \mu(t))=\sup \left\{\langle u, \mu\rangle: u \in L_{\infty}^{2}(\Omega), u^{\top}(t) w \leq r(t, w) \forall w \in \mathbb{E}^{2}\right\}
$$

where $\langle\cdot, \cdot\rangle$ denotes the duality pairing on $L_{\infty}^{2}(\Omega) \times L_{\infty}^{2}(\Omega)^{*}$. The function $r$ is the local cost rate and $\alpha$ is the common distribution of supply and demand. A "transportation flow" $\mu$ is an additive set function of bounded variation on $\mathfrak{B}$. For details of the model we refer to [4] and the references given there. The dual problem to (3.1)-(3.3) has the following form:

Maximize

$$
\int_{\Omega} u(t) d \alpha(t)
$$

subject to

$$
u \in W_{\infty}^{1}(\Omega)
$$

and

$$
\nabla^{\top} u(t) w \leq r(t, w) \quad \text { a.e. in } \Omega \text { and for all } w \in \mathbb{E}^{2} .
$$

We shall not deal here with this general case. Instead we make some specializations:

First: We consider the case of a local cost rate depending only on the location but not on the direction of the flow. This means that $r(t, w)$ has the form $r(t, w)=\tilde{r}(t)|w|$. For simplicity of notations we shall replace $r(t, w)$ with $r(t)|w|$. The above assumptions on $r$ read then:

- $r(\cdot)$ is summable on $\Omega$.

- $\gamma_{1} \leq r(t) \leq \gamma_{2}$ for all $t \in \Omega$ with some positive constants $\gamma_{1}, \gamma_{2}$.

Second: We consider a measure $\alpha$ which is the difference of a finitely-discrete and an absolutely continuous measure as in the Kantorović-Monge problem.

Now, if a transport from a supply point $T_{i}$ to some point $t^{\prime} \in \Omega$ occurs (in the above terminology), then it always takes the shortest path in the "density field" defined by 
the function $r$. (Among all curves $\mathfrak{C}$ connecting $T_{i}$ and $t^{\prime}$ this is the curve along which $\int_{\mathbb{C}} r(t) d t$ is minimal.)

With these specializations, the constraints (3.8) of the dual problem are turned into

$$
\nabla^{\top} u(t) w \leq r(t) \quad \text { a.e. in } \Omega \text { and for all } w \in \mathbb{E}^{2},|w|=1 \text {. }
$$

Since the right-hand sides of the inequalities in (3.9) do not depend on $w$, this is equivalent to

$$
|\nabla u(t)| \leq r(t) \quad \text { a.e. in } \Omega .
$$

Now let us return to the Kantorovič-Monge problem. The constraints (2.3)-(2.4) may be replaced with

$$
\psi(e, \bar{\Omega})-\psi(\bar{\Omega}, e)=\alpha(e) \quad \text { for all } e \in \mathfrak{B}
$$

where $\alpha=\phi_{0}-\phi_{1}$ is now the common distribution of supply and demand. The feasible domain described by (2.2)-(2.4) is a proper subset of the feasible domain described by (2.2), (3.11): If $\psi$ satisfies (2.3)-(2.4), then it also satisfies (3.11). And if additionally the measure $\psi_{0} \geq 0$ satisfies

$$
\psi_{0}(e, \bar{\Omega})-\psi_{0}(\bar{\Omega}, e)=0 \quad \text { for all } e \in \mathfrak{B}
$$

then $\psi+\psi_{0}$ obviously satisfies (3.11), but not necessarily (2.3) and (2.4). ( $\psi_{0}$ could, by analogy to flow problems on graphs, be called a circulation.) But the optimal values of the problems (2.1)-(2.4) and (2.1)-(2.2), (3.11) are the same and an optimal solution to (2.1)-(2.2), (3.11) necessary fulfills (2.3)-(2.4).

The dual problem to (2.1)-(2.2), (3.11) is the following:

Maximize

$$
\int_{\bar{\Omega}} u(t) d \alpha(t)
$$

subject to

$$
u \in C(\bar{\Omega})
$$

and

$$
u(t)-u\left(t^{\prime}\right) \leq\left|t-t^{\prime}\right| \quad \text { for all } t, t^{\prime} \in \bar{\Omega} .
$$

Because of the symmetry of this inequality it follows that

$$
\left|u(t)-u\left(t^{\prime}\right)\right| \leq\left|t-t^{\prime}\right| \quad \text { for all } t, t^{\prime} \in \bar{\Omega} .
$$

This means that every feasible solution to the dual problem is a. Lipschitz continuous function with Lipschitz constant 1 . But such a function is differentiable almost everywhere and its gradient (where it exists) has a norm less than or equal to the Lipschitz constant, 1 in this case. So we can replace (3.13)-(3.14) with

$$
u \in C(\bar{\Omega}), \quad \cdot u \text { differentiable a.e. in } \Omega
$$


and

$$
|\nabla u(t)| \leq 1 \quad \text { a.e. }
$$

Now, if we compare the dual problems (3.12), (3.15)-(3.16) and (3.6)-(3.7), (3.10), we see that they are nearly identical. The right-hand side of (3.10) is more general than that of (3.16) (setting $r(t) \equiv 1$ in (3.10) gives (3.16)), and there is the much more essential difference in the spaces in (3.15) and (3.7). This is the main reason that the primal problems cannot be compared in a similar way. But this comparison makes it clear that indeed Klötzler's transportation flow problem is a generalization of the Kantorovič-Monge problem.

\section{Numerical experiments}

We have tried three approaches to the practical solution of the transportation flow problem and its dual as described in this paper (i.e. not in its original, more general form). Setting $r$ to a constant value in $\Omega$ then gives the Kantorovič-Monge problem. In this paper, these approaches are only outlined. Details may be the subject of a subsequent publication.

The first approach is based on an idea by Klötzler. It starts from the dual problem which is replaced with a semi-infinite linear or nonlinear program. We proceed with the following steps:

- Triangulation of the domain $\Omega$ : The domain is subdivided into triangles such that the intersection of any two triangles is either an edge or a vertex or void.

- Restriction of the dual variables $u$ : The space of dual variables is replaced with the subspace of continuous functions which are affine-linear in each triangle of the triangulation. (This step is very similar to the ideas used in Finite Element Methods.)

- Replacement of the measure $\alpha$ : The total mass of each triangle $\Delta$, i.e. $\int_{\Delta} d \alpha$ is concentrated in its vertices. Then for each vertex in the triangulation these values are summed.

- Replacement of the function $r$ : The function $r$ is replaced with a constant $r_{\Delta}$ in each triangle $\Delta$ of the triangulation. As we shall see below, this must be the minimum value of $r$ in $\Delta$.

The variables of the problem are now the values of the function $u$ in the vertices of the triangulation. Because of the assumption $\int_{\Omega} d \alpha=0$ the value of the objective functional does not change if $u$ is changed by an additive constant. Thus we may fix the value of $u$ in one (arbitrarily choosen) vertex. The objective functional itself turns into a linear function of the form

$$
\sum_{i} \alpha_{i} u_{i}
$$

where the summation runs over all vertices of the triangulation, $u_{i}$ denotes the value of $u$ in the $i$-th vertex and $\alpha_{i}$ is the replacement value for $\alpha$. Since the gradient of an affine-linear function is constant we get from (3.9)

$$
d_{j}^{\top} w \leq r(t) \quad \text { for all } t \in \Delta_{j}, w \in \mathbb{E}^{2},|w|=1
$$


for the $j$-th triangle $\Delta_{j}$. The left-hand sides of these inequalities do not depend on $t$, so we can replace the right-hand sides with the minimum value $r_{j}$ of $r$ in $\Delta_{j}$ and get

$$
d_{j}^{\top} w \leq r_{j} \quad \text { for all } w \in \mathbb{E}^{2},|w|=1
$$

The gradient $d_{j}=\nabla u$ of $u$ in the triangle $\Delta_{j}$ may be easily expressed in terms of the values $u_{i}$ in the vertices forming this triangle.

As a consequence, the dual problem is replaced with a semi-infinite linear program: The number of variables is finite (equal to the number of vertices in the triangulation), the objective function (4.1) is linear, and for each triangle $\Delta_{j}$ in the triangulation we have an infinite set of linear constraints of the form (4.2). The solution of such a problem is in principle not problematic. Since we are interested in the solution of the primal problem as well its seems reasonable to use a variant of the semi-infinite simplex algorithm as described in $[10,11]$ (for a short description, see also [5]). But in the concrete situation the semi-infinite simplex algorithm does not work satisfactorily. The reason is that in each step of the algorithm a finite subproblem of (4.1)-(4.2) is solved by the dual simplex algorithm which leads to an optimal basic feasible solution to the dual of this subproblem. Unfortunately, the dual to the dual problem (4.1)-(4.2) (which should be considered as a replacement for the primal transportation flow problem) has no optimal basic feasible solution. As a consequence, the basis matrices of the partial problems tend to singularity.

The equivalence of (3.9) and (3.10) suggests another approach based on the same discretization: to maximize (4.1) subject to the finite number of nonlinear constraints

$$
\left|d_{j}\right|^{2} \leq r_{j}^{2}
$$

The problem (4.1), (4.3) has a linear objective function and a finite number of nonlinear (but convex) inequality constraints. It can be solved, e.g., with an SQP method. As starting point we may use the last solution of (4.1)-(4.2) found with the semi-infinite simplex algorithm.

The third approach is based on an idea by DeweB (cf. [2]). The primal problem is replaced with a minimum cost flow problem on a suitably constructed graph. We proceed with the following steps:

- Construction of a grid over $\Omega$ : The domain $\Omega$ is covered with a regular grid. For simplicity, we assume that all supply points $T_{i}$ are nodes of the grid.

- Forming of a complete bidirectional graph: Temporarily, we build a graph with the above grid as the node set and arcs in both directions between all pairs of nodes.

- Weighting the arcs of the graph: The arcs of the graph are weighted with the integral of $r(t)$ along the straight line connecting the end nodes of the arc. This integral is computed approximately by a simple quadrature.

- Reduction of the graph: Within this graph we compute, for all supply points, the trees of shortest paths to all other nodes. The graph is then reduced to the union of all these trees. 
- Distribution of supply and demand: Each node corresponding to a supply point is assigned the supply in this point. All other nodes become sinks. The demand distribution is concentrated in the sinks in such a way that supply and demand in the graph are balanced.

As result we have a directed weighted graph with sources in the supply points and sinks distributed uniformly over $\Omega$. In this graph, we solve the minimum cost flow problem. The resulting optimal flow can directly be interpreted as an approximate solution to the transportation flow problem.

Numerical experiments were done with a rather simple setting: The domain $\bar{\Omega}$ is a rectangle and the triangulation used in the first and second approaches is regular. With the available hardware it was possible to solve problems with triangulations up to approximately 500 vertices and 850 triangles (semi-infinite linear programming and nonlinear programming approaches) and graphs with approximately 2000 nodes. The results are comparable but the computation times are not. Whereas the solution of a nonlinear programming problem with 475 variables and 864 quadratic constraints took about 25 hours on a Pentium 100 PC, the solution of the minimum cost flow problem on a graph with 1728 nodes and 156,000 arcs required only about 40 minutes. (These are the data of the examples given below, for other problems the relationships were similar.). So the conclusion is (for the moment) that the third approach is clearly superior.

As an illustration, we give two examples. For both, the domain $\bar{\Omega}$ is the rectangle $[0,4] \times[0,3]$. There are two supply points located at $T_{1}=\left(\frac{2}{3}, \frac{1}{2}\right)$ and $T_{2}=\left(2, \frac{5}{3}\right)$ with equal supply $a_{1}=a_{2}=6$. The demand distribution is constant: $b(t)=1$ for all $t \in \Omega$.
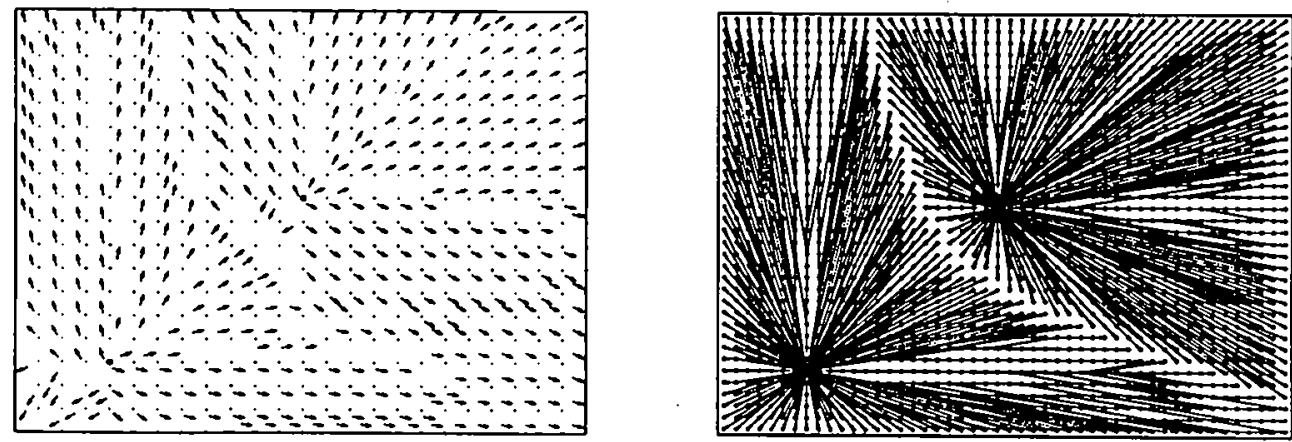

Figure 1: Kantorovič-Monge problem

The first example (Figure 1) is a Kantorovič-Monge problem, i.e. the cost rate $r(t)$ is constant and equal to 1 . This problem could be solved explicitely. The border line between the districts $\Omega_{1}$ and $\Omega_{2}$ is the hyperbola with focusses in $T_{1}$ and $T_{2}$ that divides $\Omega$ into two parts of equal area.

The second example (Figure 2) is a Klötzler transportation flow problem with nonconstant cost rate $r(t)$ : It is set to a larger value $(r(t)=10)$ along the line connecting the points $(2,0)$ and $(4,2)$ on the boundary of $\Omega$, except for a small gap at the point $(3,1)$. In contrast to the first problem, an explicit solution for the second problem is 
not possible. It is not even clear whether the "free" boundary between the districts is again a hyperbola.
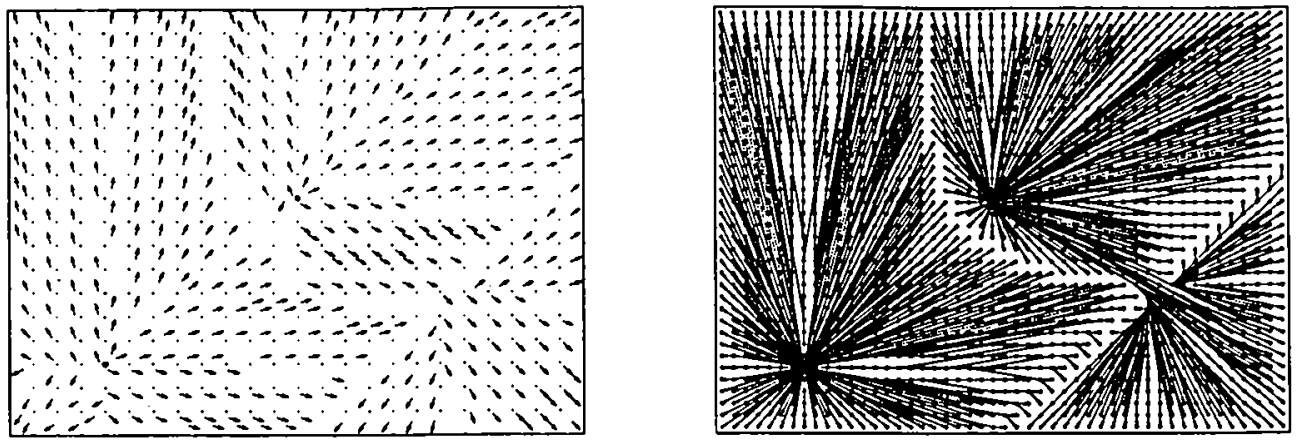

Figure 2: Transportation flow problem

(Both in Figure 1 and Figure 2, the left picture shows the flow obtained from the solution via nonlinear programming, the right picture shows the flow obtained from the minimum cost flow problem.)

Acknowledgement. Most of the computations were done with free software. The author wishes to express his thanks to all who make their software freely available for academic purposes. The following packages were used:

DSPLP from the SLATEC library (authors R. J. Hanson and K. L. Hiebert) for linear programming, FFSQP (authors J. L. Zhou, A. L. Tits, and C. T. Lawrence) for nonlinear programming, RELAX (authors D. Bertsekas, and P. Tseng - with a small modification to allow non-integer arc weights) for network flow problems, and PGPLOT (author T. J. Pearson) for plotting.

\section{References}

[1] Corley, H. W., and S. D. Roberts: A partitioning problem with applications in regional design. Operations Res. 20 (1972), $1010-1019$.

[2] DeweB, G. and P. Helbig: Motorway junctions as Steiner points in a density field and generalizations. Optimization 38 (1996), 357 - 365.

[3] Kantorovič, L. V.: On the translocation of masses (in Russian). Dokl. Acad. Nauk SSSR 37 (1942), 227 - 229.

[4] Klötzler, R.: Flußoptimierung. Z. Ang. Math. Mech. (ZAMM) 74 (1994), T591 - T593.

[5] Kosmol, P.: Methoden zur numerischen Behandlung nichtlinearer Gleichungen und Optimierungsaufgaben. Stuttgart: B. G. Teubner 1993.

[6] Levin, V. L.: Duality and approximation in the problem of translocation of masses (in Russian). In: Mathematical Economics and Functional Analysis. Moscow: Nauka 1974, $94-108$.

[7] Lowe, T. J. and A. P. Hurter: The generalized market area problem. Management Sci. 22 (1976), $1105-1115$. 
[8] Rubinšteǐn, G. S.: Duality in mathematical programming and some problems of convex analysis (in Russian). Usp. Mat. Nauk 25 (1970), 171 - 200.

[9] Rudolph, H.: Kapazitierte lineare Programme in Funktionenräumen. Beitr. Anal. 6 (1974), 155 - 168.

[10] Rudolph, H.: Der Simplexalgorithmus der semiinfiniten linearen Optimierung. Wiss. Z. Techn. Hochsch. Merseburg 29 (1987), $782-806$.

[11] Voigt, H.: Ein Beitrag zur Theorie der semiinfiniten linearen Optimierung. Dissertation. Universität Leipzig 1979.

Received 10.11.1997 